TITLE:

\title{
Classical correlation-length exponent in the nonuniversal quantum phase transition of a diluted Heisenberg antiferromagnet
}

\section{$\operatorname{AUTHOR}(\mathrm{S}):$}

Yasuda, C.; Todo, S.; Harada, K.; Kawashima, N.; Miyashita, S.; Takayama, $\mathrm{H}$.

\section{CITATION:}

Yasuda, C....[et al]. Classical correlation-length exponent in the nonuniversal quantum phase transition of a diluted Heisenberg antiferromagnet. Physical Review B 2001, 63(14): 140415.

\section{ISSUE DATE:}

2001-03-23

URL:

http://hdl.handle.net/2433/200792

RIGHT:

(C)2001 The American Physical Society 


\title{
Classical correlation-length exponent in the nonuniversal quantum phase transition of a diluted Heisenberg antiferromagnet
}

\author{
C. Yasuda, ${ }^{1}$ S. Todo, ${ }^{1, *}$ K. Harada,${ }^{2}$ N. Kawashima, ${ }^{3}$ S. Miyashita,${ }^{4}$ and H. Takayama ${ }^{1}$ \\ ${ }^{1}$ Institute for Solid State Physics, University of Tokyo, Kashiwa 277-8581, Japan \\ ${ }^{2}$ Department of Applied Analysis and Complex Dynamical Systems, Kyoto University, Kyoto 606-8501, Japan \\ ${ }^{3}$ Department of Physics, Tokyo Metropolitan University, Tokyo 192-0397, Japan \\ ${ }^{4}$ Department of Applied Physics, University of Tokyo, Tokyo 113-8656, Japan
}

(Received 25 October 2000; published 23 March 2001)

\begin{abstract}
Critical behavior of the quantum phase transition of a site-diluted Heisenberg antiferromagnet on a square lattice is investigated by means of the quantum Monte Carlo simulation with the continuous-imaginary-time loop algorithm. Although the staggered spin-correlation function decays in a power law with the exponent definitely depending on the spin size $S$, the correlation-length exponent is classical, i.e., $\nu=4 / 3$. This implies that the length scale characterizing the nonuniversal quantum phase transition is nothing but the mean size of connected spin clusters.
\end{abstract}

DOI: 10.1103/PhysRevB.63.140415

PACS number(s): 75.10.Jm, 75.10.Nr, 75.40.Cx, 75.40.Mg

Ground-state phase transitions in two-dimensional (2D) diluted quantum Heisenberg antiferromagnets (HAF's) have attracted much interest because they are caused by the coexistence of quantum fluctuations and randomness. ${ }^{1-7}$ Numerical works ${ }^{8,9}$ and theoretical works ${ }^{10,11}$ have given various estimates of the critical concentration of the system with $S$ $=1 / 2$. All of them are above the purely-geometrical percolation threshold on a square lattice, $p_{\mathrm{cl}}=0.5927460(5) .{ }^{12}$ This suggests that the phase transition could be seriously affected by quantum fluctuations.

Recently, Kato et al. ${ }^{13}$ have investigated the diluted 2D HAF with $S=1 / 2,1,3 / 2$, and 2 by means of the quantum Monte Carlo (QMC) method with the continuous-imaginarytime loop algorithm. ${ }^{14-17}$ Their conclusion is qualitatively different from the above mentioned results: the critical concentration coincides with $p_{\mathrm{cl}}$ and does not depend on $S$. The coincidence of the critical concentration with $p_{\mathrm{cl}}$ has also been reported on the bond-diluted HAF. ${ }^{18}$

The critical exponents of the phase transition at $p_{\mathrm{cl}}$ have also been estimated. Kato et al. have obtained the critical exponent $\beta$ of the zero-temperature staggered magnetization. Interestingly, the value of $\beta$ is different from that of the classical $(S=\infty)$ exponent and depends on $S$. They have also estimated other critical exponents by the finite-size scaling (FSS) analysis assuming the following form for the static staggered structure factor at zero temperature:

$$
S_{\mathrm{s}}(L, 0, p) \sim L^{\Psi} \widetilde{S}_{\mathrm{s}}\left[L^{1 / v}\left(p-p_{\mathrm{cl}}\right)\right],
$$

where $L$ is the system size and

$$
S_{\mathrm{s}}(L, T, p) \equiv \frac{1}{L^{d}} \sum_{i, j} \mathrm{e}^{\mathrm{i} \vec{k} \cdot\left(\vec{r}_{i}-\vec{r}_{j}\right)}\left\langle S_{i}^{z} S_{j}^{z}\right\rangle
$$

with $\vec{k}=(\pi, \pi)$ and $d=2$. The bracket $\langle\ldots\rangle$ in Eq. (2) denotes both the thermal and random averages. The scaling function $\widetilde{S}_{\mathrm{s}}(x)$ in Eq. (1) has the following asymptotic form:

$$
\widetilde{S}_{\mathrm{s}}(x) \sim\left\{\begin{array}{lll}
x^{2 \beta} & \text { for } & x \gg 1 \\
|x|^{-\nu \Psi} & \text { for } & x \ll-1,
\end{array}\right.
$$

where the exponent $\beta$ is related to $\Psi$ and $\nu$ by the scaling relation

$$
2 \beta=(d-\Psi) \nu .
$$

They have estimated the critical exponent $\Psi$ by the FSS analysis exactly at $p=p_{\mathrm{cl}}$ and have found that it depends on $S$.

Kato et al. have attributed the $S$ dependence in exponent $\beta$ or $\Psi$ to quantum fluctuations, while the length exponent $\nu$ is assumed to be given by the classical one, $\nu_{\mathrm{cl}}=4 / 3,{ }^{19}$ which governs a power-law divergence of the mean size of connected spin clusters as $\lambda(p) \propto\left|p-p_{\mathrm{cl}}\right|^{-\nu_{\mathrm{cl}}}$. Namely, they have assumed that the staggered spin correlation function between two spins in a cluster is described by the scaling expression

$$
C(i, j ; p) \sim r_{i, j}^{-\alpha} \widetilde{C}\left[r_{i, j} / \lambda(p)\right],
$$

with $\widetilde{C}(x) \sim$ const. at $x \ll 1$. The power-law decay of the correlation function is due to quantum fluctuations and its $S$-dependent exponent $\alpha$ is related to $\Psi$ by

$$
\Psi=2 D-d-\alpha,
$$

where $D$ is the fractal dimension $(91 / 48$ for $d=2)$. Kato et $a l$. have checked this scenario simply by evaluating $\nu$ through Eq. (4) using $\beta$ and $\Psi$ obtained by their simulation.

In the present paper, we perform the FSS analysis on $S_{\mathrm{s}}(L, 0, p)$ of systems with $S=1 / 2$ and 1 more systematically by carrying out the QMC simulation at various concentrations not only at $p_{\mathrm{cl}}$. It is found, as we see below, that our QMC data are well described by the FSS form of Eq. (1) in a whole range of $p$ studied, which includes the asymptotic ranges $x \gg 1$ and $x \ll-1$ in Eq. (3). Within numerical accuracy of our analysis, exponent $\Psi$ turns out to definitely depend on the spin size $S$, while exponent $\nu$ does not. The latter 

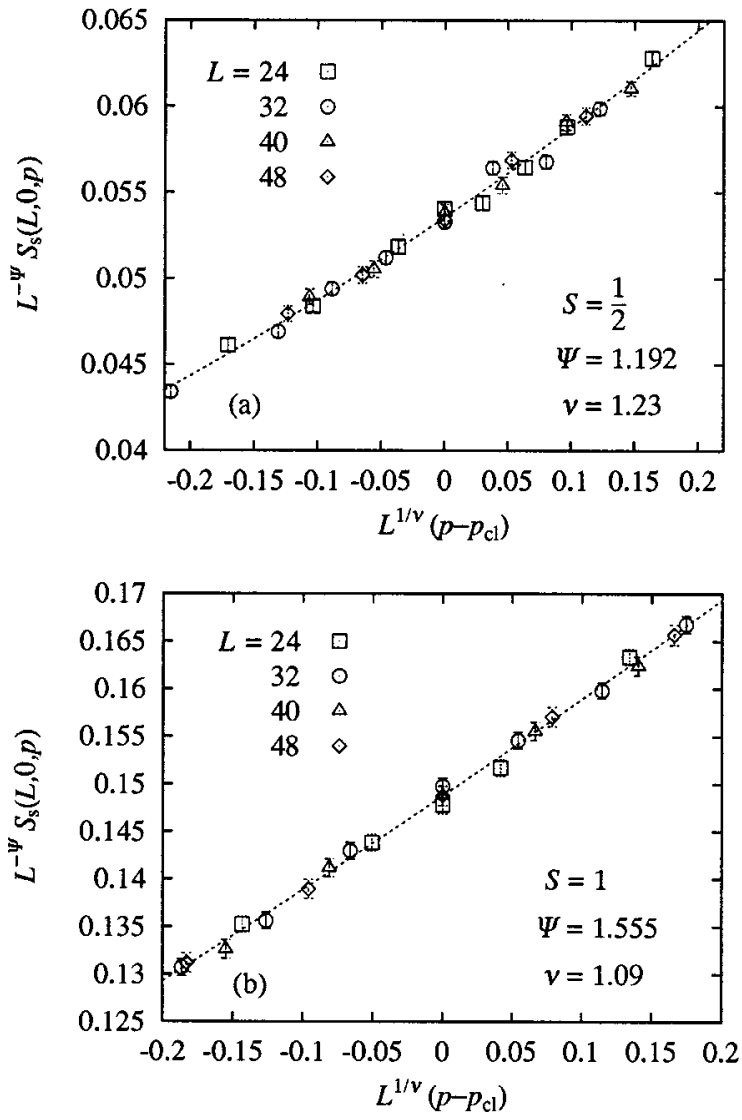

FIG. 1. Scaling plot of $S_{\mathrm{s}}(L, 0, p)$ for (a) $S=1 / 2$ and (b) $S=1$. The dashed line represents $\widetilde{S}_{\mathrm{s}}(x)$, which is approximated by a polynomial of order 2 .

$S$-independent value of $\nu$ coincides with the classical one $(=4 / 3)$ within the error bar. These results further support the above mentioned scenario of the quantum phase transition in the diluted 2D HAF, particularly, the ansatz that the length scale characterizing the transition is nothing but the mean size of connected spin clusters.

The system we study is the site-diluted HAF on a square lattice described by the Hamiltonian

$$
\mathcal{H}=J \sum_{\langle i, j\rangle} \epsilon_{i} \epsilon_{j} \mathbf{S}_{i} \cdot \mathbf{S}_{j}
$$

where $J(>0)$ is the antiferromagnetic coupling constant, $\Sigma_{\langle i, j\rangle}$ denotes the summation over all nearest-neighbor pairs and $\mathbf{S}_{i}$ is the quantum spin operator at site $i$. The quenched magnetic occupation factors $\left\{\epsilon_{i}\right\}$ independently take 1 or 0 with probability $p$ and $1-p$, respectively. We simulate $L$ $\times L$ square lattices with the periodic boundary condition by means of the same QMC method with the continuousimaginary-time loop algorithm as that adopted by Kato et al. An improved estimator is used to calculate the static staggered structure factor. At each parameter set $(L, T, p)$ physical quantities of interest are averaged over 1000-3000 samples. At each sample, $10^{3}-10^{4}$ Monte Carlo steps (MCS) are spent for measurement after $500-10^{3}$ MCS for thermalization.

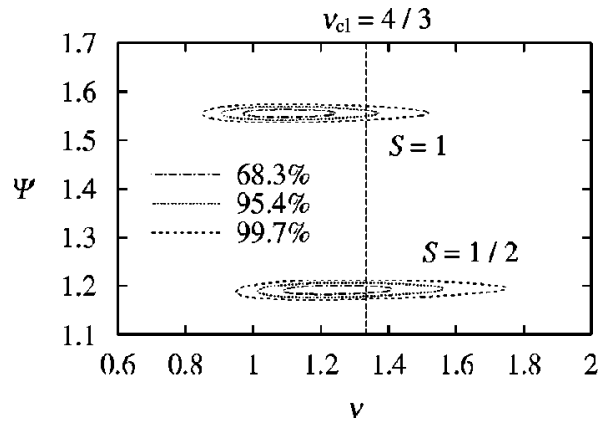

FIG. 2. Confidence region of $\Psi$ and $\nu$. The percentages $68.3 \%$ $(1-\sigma), 95.4 \%(2-\sigma)$, and $99.7 \%(3-\sigma)$ represent the probability that the true parameter values fall within the confidence regions. The classical value of $\Psi$ is 1.79167 . The vertical dashed line indicates the classical value of $\nu, 4 / 3$.

In a finite system, $S_{\mathrm{s}}(L, T, p)$ converges rapidly to its zero-temperature values at temperatures lower than the gap due to the finiteness of the system. The saturation temperature turns out to be smaller for smaller $\left|p-p_{\mathrm{cl}}\right|$. In the present work $S_{\mathrm{s}}(L, 0, p)$ close to $p_{\mathrm{cl}}$ is approximated by $S_{\mathrm{s}}(L, T, p)$ at the following temperatures: for the $S=1 / 2$ system $T=0.002 J$ for $L=24$ and $T=0.001 J$ for $L=32,40$, and 48, and for the $S=1$ system $T=0.01 J$ for $L=24$ and $T$ $=0.005 \mathrm{~J}$ for $L=32,40$, and 48 .

Let us first examine the FSS analysis of $S_{\mathrm{s}}(L, 0, p)$ at $p$ close to $p_{\mathrm{cl}}$. The results of the $S=1 / 2(S=1)$ system at $0.580 \leqslant p \leqslant 0.605(0.585 \leqslant p \leqslant 0.600)$ are shown in Figs. 1(a) and 1(b). The error bars in the figures represent the standard deviation. In the FSS fit the critical concentration is set to be $p_{\mathrm{cl}}(\simeq 0.5927460)$, and the scaling function $\widetilde{S}_{\mathrm{s}}(x)$ is approximated by a polynomial of order 2 . As seen in Fig. 1(a), the QMC data for $S=1 / 2$ are well scaled with $\Psi=1.192$ and $\nu$ $=1.23$. The statistical accuracy of the fit is shown in Fig. 2, where we draw the confidence region within which the true values of $\Psi$ and $\nu$ fall with probability $68.3 \%(1-\sigma), 95.3 \%$ $(2-\sigma)$, or $99.7 \%(3-\sigma)$. Similarly, the data for $S=1$ are well scaled with $\Psi=1.555$ and $\nu=1.09$, as seen in Figs. 1(b) and 2 . From these results we can conclude that exponent $\Psi$ definitely depends on the spin size $S$. The exponents $\nu$ of $S$ $=1 / 2$ and 1 , on the other hand, coincide with each other and with its classical value $(=4 / 3)$ within the numerical accuracy of the present analysis. The obtained values of $\Psi, \nu, \alpha$, and $\beta$ are summarized in Table I. The exponents $\alpha$ and $\beta$ are calculated by the scaling relations, Eqs. (4) and (6).

The value of $\Psi$ for $S=1 / 2$ obtained above is consistent

TABLE I. Summary of critical exponents $\Psi, \nu, \alpha$, and $\beta$ for $S=1 / 2,1$ and the classical case $(S=\infty)$. The values of $\Psi$ and $\nu$ are obtained by the FSS analysis shown in Figs. 1(a) and 1(b), and $\alpha$ is calculated as $\alpha=2 D-d-\Psi$, and $\beta$ as $\beta=(d-\Psi) \nu / 2$.

\begin{tabular}{ccccc}
\hline \hline$S$ & $\Psi$ & $\nu$ & $\alpha$ & $\beta$ \\
\hline $1 / 2$ & $1.192(9)$ & $1.23(16)$ & $0.600(9)$ & $0.50(7)$ \\
1 & $1.555(8)$ & $1.09(14)$ & $0.237(8)$ & $0.24(4)$ \\
$\infty$ & 1.79167 & 1.33333 & 0. & 0.13889 \\
\hline
\end{tabular}


with the one $[=1.17(6)]$ that has been estimated by Kato et $a l$. from $S_{\mathrm{s}}(L, 0, p)$ at $p=p_{\mathrm{cl}}$. In their analysis $S_{\mathrm{s}}\left(L, 0, p_{\mathrm{cl}}\right)$ is approximated by $S_{\mathrm{s}}\left(L, T, p_{\mathrm{cl}}\right)$ at low temperatures where its $T$ dependence becomes nondiscernible within the error bars. Kato et al. have also performed the FSS analysis making use of the data at all temperatures they have simulated. This analysis yields $\Psi=1.27(2)$ which differs distinctly from the present result listed in Table I. This discrepancy may be due to the systematic error in our estimates where $S_{\mathrm{s}}(L, 0, p)$ is approximated by $S_{\mathrm{s}}(L, T, p)$ at a small but finite temperature as described before. It should be remedied when the finitetemperature FSS analysis is carried out as done by Kato et al.

From Fig. 2 one sees that the accuracy of $\nu$ is significantly poorer than that of $\Psi$, which is interpreted as follows. The value of $\Psi$ can be essentially extracted solely from $S_{\mathrm{s}}\left(L, 0, p_{\mathrm{cl}}\right)$, while to evaluate $\nu$ we need $S_{\mathrm{s}}(L, 0, p)$ at $p$ other than $p_{\mathrm{cl}}$, or $\delta S_{\mathrm{s}}(L, 0, p) / \delta p$ at $p=p_{\mathrm{cl}}$. Naturally, the statistical error of $\nu$ is expected to be larger than that of $\Psi$. Concerned with the systematic error in $\nu$ arising from finitetemperature corrections, on the other hand, the saturation temperature of $S_{\mathrm{s}}(L, T, p)$ becomes smaller for smaller $\left|p-p_{\text {cl }}\right|$, i.e., the effect of finite-temperature corrections to $S_{\mathrm{s}}(L, T, p)$ becomes maximal exactly at $p=p_{\mathrm{cl}}$. This implies that the leading order finite-temperature correction to $\delta S_{\mathrm{s}}(L, 0, p) / \delta p$ at $p=p_{\mathrm{cl}}$ disappears, and so the systematic error in $\nu$ is much smaller than that in $\Psi$.

Next let us discuss $S_{\mathrm{s}}(L, 0, p)$ in the full range of $p$ we have simulated, i.e., $0.2 \leqslant p \leqslant 1$, which is much wider than that in Fig. 1. As shown in Figs. 3(a) and 3(b), respectively, for $S=1 / 2$ and 1, all QMC data turn out to lie on a universal curve when $L^{-\Psi} S_{\mathrm{s}}(L, 0, p)$ are plotted against $L^{1 / \nu}\left|p-p_{\mathrm{cl}}\right|$ by using the exponents $\Psi$ and $\nu$ listed in Table I. For $L^{1 / \nu}\left|p-p_{\mathrm{cl}}\right|>1$, the data points merge to the dashed line, which represents $a x^{2 \beta}$ for $p>p_{\mathrm{cl}}$ and $b|x|^{-\nu \Psi}$ for $p<p_{\mathrm{cl}}$, where $\beta, \nu$, and $\Psi$ are those listed in Table $\mathrm{I}$, and $a$ and $b$ are arbitrary constants adjusted to fix the position of the asymptotic lines. At much larger $L^{1 / \nu}\left|p-p_{\mathrm{cl}}\right|$ the scaling fit becomes deteriorated, indicating that the corresponding $p$ is out of the scaling region. The results shown in Fig. 3 also support the scenario due to Kato et al. on the nonuniversal quantum phase transition.

In summary, in order to establish nature of the nonuniversal quantum phase transition of 2D site-diluted HAF's for $S=1 / 2$ and 1 , we have performed the QMC simulation in a relatively wider region of concentration than that of Kato et $a l$. and have estimated the critical exponent $\nu$ more systematically. We have observed that the static staggered structure factor is well described by the scaling form of Eq. (1) with Eq. (3). In particular, the exponent $\nu$ is confirmed to coincide with the classical one. These results support the following
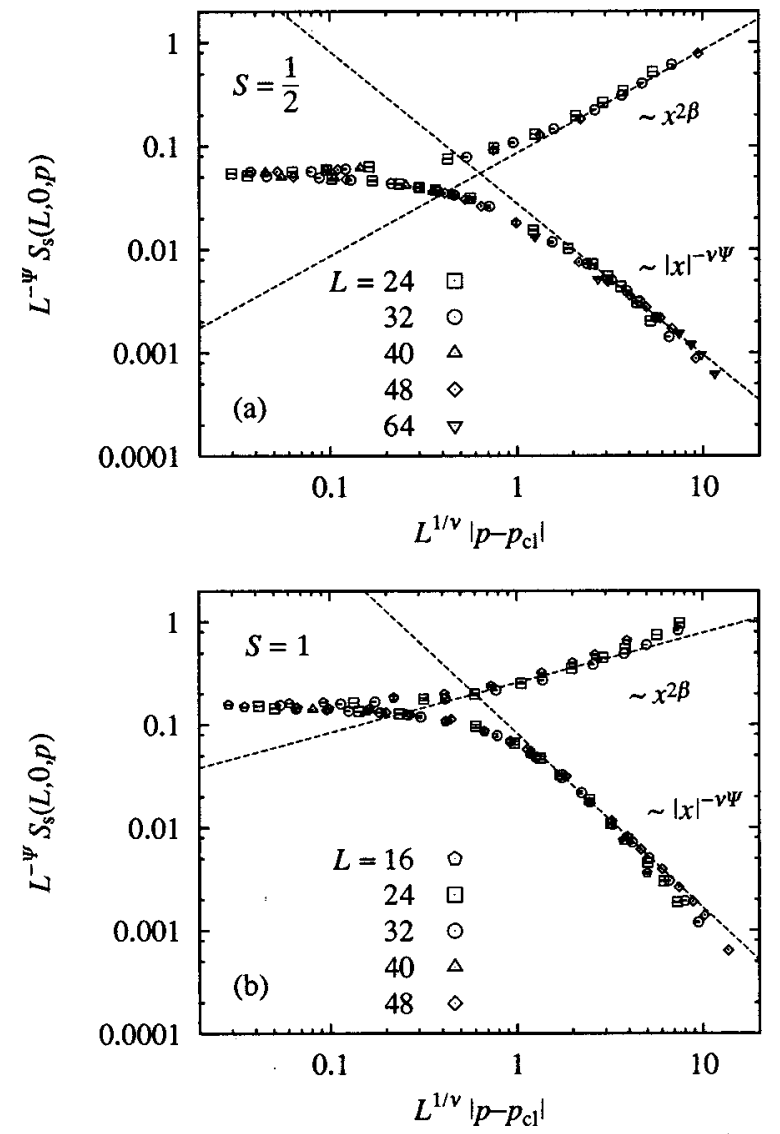

FIG. 3. The double-logarithmic plot of $L^{-\Psi} S_{\mathrm{s}}(L, 0, p)$ against $L^{1 / \nu}\left|p-p_{\text {cl }}\right|$ for (a) $S=1 / 2$ and (b) $S=1$. Dashed lines represent $a x^{2 \beta}$ and $b|x|^{-\nu \Psi}$.

arguments by Kato et al.: (1) there exists no other macroscopic characteristic lengths than $\lambda(p)$, the mean size of connected spin clusters at concentration $p$, and (2) the staggered spin correlation function between two sites on a fractal cluster decays in a power law as $C(i, j ; p) \sim r_{i, j}^{-\alpha}$, where $\alpha$ depends on the strength of quantum fluctuations specified by the spin size $S$.

Most of the numerical calculations for the present work have been performed on the CP-PACS at the University of Tsukuba, the Hitachi SR-2201 at the Supercomputer Center, University of Tokyo, and the SGI2800 at the Institute for Solid State Physics, University of Tokyo. The present work was supported by the "Large-scale Numerical Simulation Program" of the Center for Computational Physics, University of Tsukuba, and also by the "Research for the Future Program" (JSPS-RFTF97P01103) of the Japan Society for the Promotion of Science.
*Present address: Theoretische Physik, Eidgenössische Technische Hochschule, CH-8093 Zürich, Switzerland.

${ }^{1}$ D. J. Breed, K. Gilijamse, J. W. E. Sterkenburg, and A. R. Miedema, Physica 68, 303 (1973).

${ }^{2}$ L. J. de Jongh, in Magnetic Phase Transitions, edited by M. Aus- loos and R. J. Elliott (Springer-Verlag, Berlin, 1983), p. 172.

${ }^{3}$ A. Chakraborty, A. J. Epstein, M. Jarrell, and E. M. McCarron, Phys. Rev. B 40, 5296 (1989).

${ }^{4}$ S-W. Cheong, A. S. Cooper, L. W. Rupp, Jr., B. Batlogg, J. D. Thompson, and Z. Fisk, Phys. Rev. B 44, 9739 (1991). 
${ }^{5}$ S. T. Ting, P. Pernambuco-Wise, J. E. Crow, E. Manousakis, and J. Weaver, Phys. Rev. B 46, 11772 (1992).

${ }^{6}$ S. J. Clarke and A. Harrison, J. Phys.: Condens. Matter 4, 6217 (1992); J. Magn. Magn. Mater. 140-144, 1627 (1995).

${ }^{7}$ M. Corti, A. Rigamonti, F. Tabak, P. Carretta, F. Licci, and L. Raffo, Phys. Rev. B 52, 4226 (1995).

${ }^{8}$ J. Behre and S. Miyashita, J. Phys. A 25, 4745 (1992); S. Miyashita, J. Behre, and S. Yamamoto, in Quantum Monte Carlo Methods in Condensed Matter Physics, edited by M. Suzuki (World Scientific, Singapore, 1994), p. 97.

${ }^{9}$ A. W. Sandvik and M. Vekić, Phys. Rev. Lett. 74, 1226 (1995).

${ }^{10}$ C. Yasuda and A. Oguchi, J. Phys. Soc. Jpn. 66, 2836 (1997); 68, 2773 (1999).

${ }^{11}$ Y. C. Chen and A. H. Castro Neto, Phys. Rev. B 61, R3772 (2000).

${ }^{12}$ R. M. Ziff, Phys. Rev. Lett. 69, 2670 (1992).
${ }^{13}$ K. Kato, S. Todo, K. Harada, N. Kawashima, S. Miyashita, and H. Takayama, Phys. Rev. Lett. 84, 4204 (2000).

${ }^{14}$ H. G. Evertz, cond-mat/9707221 (unpublished).

${ }^{15}$ H. G. Evertz, G. Lana, and M. Marcu, Phys. Rev. Lett. 70, 875 (1993); U.-J. Wiese and H.-P. Ying, Z. Phys. B: Condens. Matter 93, 147 (1994); B. B. Beard and U.-J. Wiese, Phys. Rev. Lett. 77, 5130 (1996); N. Kawashima and J. E. Gubernatis, ibid. 73, 1295 (1994).

${ }^{16}$ K. Harada, M. Troyer, and N. Kawashima, J. Phys. Soc. Jpn. 67, 1130 (1998).

${ }^{17}$ S. Todo and K. Kato, cond-mat/9911047 (unpublished).

${ }^{18}$ A. W. Sandvik, cond-mat/9909230 (unpublished).

${ }^{19}$ D. Stauffer, Phys. Rep. 54, 1 (1979); D. Stauffer and A. Aharony, An Introduction to Percolation Theory, 2nd ed. (Taylor and Francis, London, 1985), and references therein. 